\title{
Identification of the molecular regulation of differences in lipid deposition in dedifferentiated preadipocytes from different chicken tissues
}

Zheng Ma ${ }^{1+}$, Na Luo ${ }^{2 \dagger}$, Lu Liư ${ }^{2}$, Huanxian Cui ${ }^{2}$, Jing Li ${ }^{1}$, Hai Xiang ${ }^{1}$, Huimin Kang ${ }^{1}$, Hua Li ${ }^{*}$ and Guiping Zhao ${ }^{1,2^{*}}$

\begin{abstract}
Background: A body distribution with high intramuscular fat and low abdominal fat is the ideal goal for broiler breeding. Preadipocytes with different origins have differences in terms of metabolism and gene expression. The transcriptome analysis performed in this study of intramuscular preadipocytes (DIMFPs) and adipose tissue-derived preadipocytes (DAFPs) aimed to explore the characteristics of lipid deposition in different chicken preadipocytes by dedifferentiation in vitro.
\end{abstract}

Results: Compared with DAFPs, the total lipid content in DIMFPs was reduced $(P<0.05)$. Moreover, 72 DEGs related to lipid metabolism were screened, which were involved in adipocyte differentiation, fatty acid transport and fatty acid synthesis, lipid stabilization, and lipolysis. Among the 72 DEGs, 19 DEGs were enriched in the PPAR signaling pathway, indicating its main contribution to the regulation of the difference in lipid deposition between DAFPs and DIMFPs. Among these 19 genes, the representative APOA1, ADIPOQ, FABP3, FABP4, FABP7, HMGCS2, LPL and RXRG genes were downregulated, but the ACSL1, FABP5, PCK2, PDPK1, PPARG, SCD, SCD5, and SLC27A6 genes were upregulated $(P<0.05$ or $P<0.01)$ in the DIMFPs. In addition, the well-known pathways affecting lipid metabolism (MAPK, TGF-beta and calcium) and the pathways related to cell communication were enriched, which may also contribute to the regulation of lipid deposition. Finally, the regulatory network for the difference in lipid deposition between chicken DAFPs and DIMFPs was proposed based on the above information.

Conclusions: Our data suggested a difference in lipid deposition between DIMFPs and DAFPs of chickens in vitro and proposed a molecular regulatory network for the difference in lipid deposition between chicken DAFPs and DIMFPs. The lipid content was significantly increased in DAFPs by the direct mediation of PPAR signaling pathways. These findings provide new insights into the regulation of tissue-specific fat deposition and the optimization of body fat distribution in broilers.

Keywords: Dedifferentiated preadipocytes, Different tissue derivation, Lipid deposition, DEGs, Chicken

\footnotetext{
* Correspondence: okhuali@fosu.edu.cn; zhaoguiping@caas.cn

'Zheng Ma and Na Luo contributed equally to this work.

'School of Life Science and Engineering, Foshan University; Guangdong

Provincial Key Laboratory of Animal Molecular Design and Precise Breeding,

Foshan 534861, China

Full list of author information is available at the end of the article
}

(c) The Author(s). 2021 Open Access This article is licensed under a Creative Commons Attribution 4.0 International License, which permits use, sharing, adaptation, distribution and reproduction in any medium or format, as long as you give appropriate credit to the original author(s) and the source, provide a link to the Creative Commons licence, and indicate if changes were made. The images or other third party material in this article are included in the article's Creative Commons licence, unless indicated otherwise in a credit line to the material. If material is not included in the article's Creative Commons licence and your intended use is not permitted by statutory regulation or exceeds the permitted use, you will need to obtain permission directly from the copyright holder. To view a copy of this licence, visit http://creativecommons.org/licenses/by/4.0/ The Creative Commons Public Domain Dedication waiver (http://creativecommons.org/publicdomain/zero/1.0/) applies to the data made available in this article, unless otherwise stated in a credit line to the data. 


\section{Background}

Fat has unique distribution characteristics and different economic values in various tissues of animals. In broilers, high-intensity artificial breeding has effectively increased the meat yield but has also increased the abdominal fat content and reduced intramuscular fat deposition [1]. Excessive abdominal fat deposition has negative impacts on the feed efficiency and carcass yield $[2,3]$. Decreased abdominal fat deposition is beneficial to reduce waste and improve consumer acceptance. In contrast, intramuscular fat is economically desirable in broiler production. Appropriately increased IMF content can improve the meat quality, including color, tenderness, flavor, and juiciness [4-7]. Lowering abdominal fat and increasing intramuscular fat can effectively increase the economic value of broilers.

Previous studies have shown that adipocytes with different origins exhibit differential differentiation capabilities [8]. Compared with subcutaneous preadipocytes, the cell size and lipid droplets in intramuscular adipocytes are smaller $[9,10]$, and the gene expression and enzyme activation related to lipid metabolism are lower in intramuscular adipocytes [11, 12]. Similarly, abdominal fat-derived preadipocytes exhibited a higher adipogenic differentiation ability than intramuscular fatderived preadipocytes in chickens $[13,14]$. However, it is still unknown whether the difference in the lipogenesis ability of preadipocytes from different tissues will disappear after cultivation in vitro.

In this study, we explored the lipogenesis characteristics of chicken preadipocytes of different origins after cultivation in vitro, including dedifferentiated intramuscular preadipocytes (DIMFPs) and dedifferentiated abdominal preadipocytes (DAFPs). These results will help to understand tissue-specific lipid deposition and optimize body fat distribution in broilers.

\section{Results}

The difference in lipid deposition in the two types of preadipocytes

Collect the DIMFP group and DAFP group cells were collected to detect the total lipid content by an Oil Red O staining assay. As shown in Fig. 1a, the total lipid content in DAFP cells was significantly $(P<0.05)$ higher than that in DIMFP cells. The main ingredients of lipids, triglycerides (TGs), phospholipids (PLIPs), and total cholesterol (TCHO) were also detected. Similarly, the TG content in DAFP cells was significantly $(P<0.05)$ higher than that in DIMFP cells. However, the contents of PLIP and TCHO showed no difference in the two types of preadipocytes (Fig. 1b).

\section{Identification of DEGs}

Total RNA of each of the three cell repetitions of the DIMFP and DAFP groups was extracted for RNA sequencing. A total of 21,469 expressed genes were found in DIMFPs and DAFPs (Additional file 1: Table S1). Using gene expression profiling and comparing the DAFP group with the DIMFP group (DIMFP vs DAFP), a total of 3629 known DEGs $\left(\left|\log _{2} \mathrm{FC}\right| \geq 1\right.$, with $\left.P<0.05\right)$ were screened (Fig. 2a), of which 2579 DEGs were downregulated and 907 DEGs were upregulated (Additional file 2: Table S2). Next, cluster analysis was performed on these 21,469 genes, and two results showed the same situation: three cell samples of the same groups were clustered together (Fig. 2b).

\section{Analysis of the enriched GO terms and pathways in the two types of preadipocytes}

Based on 3629 known DEGs, Gene Ontology (GO) analysis was performed, and 56 GO terms were enriched $(P<0.05)$, mainly including the following processes: cell adhesion, tight adhesion, cell
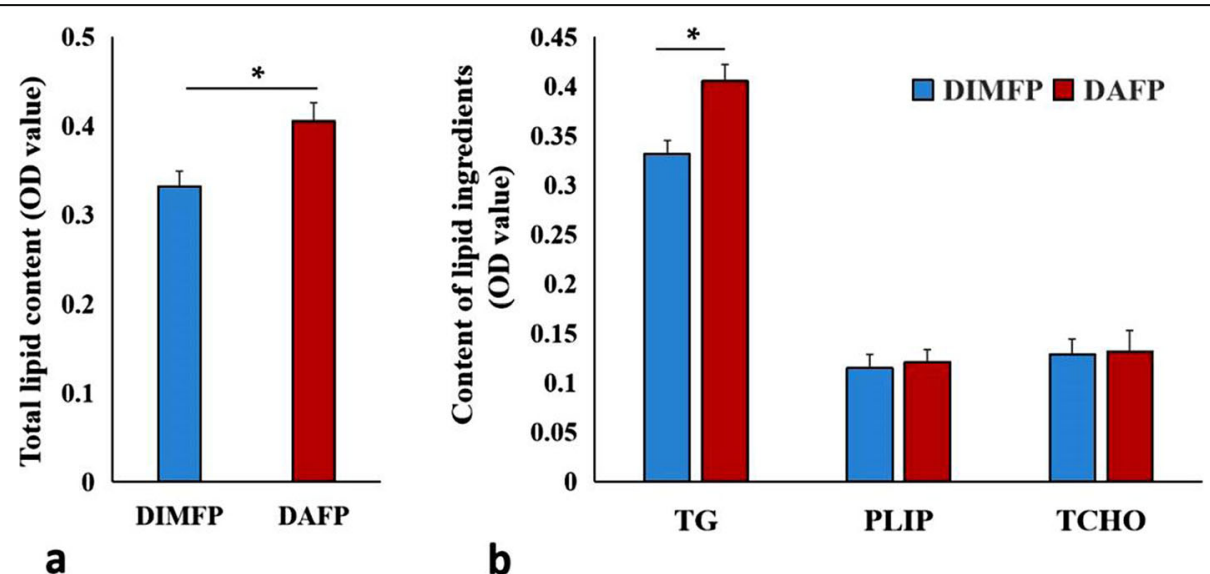

Fig. 1 Difference in lipid metabolism between DIMFPs and DAFPs of chickens. $\mathbf{a}$ and $\mathbf{b}$ The contents of total lipids and the main ingredients of lipids (TG, PLIP and TCHO). The total lipid and TG contents were increased in the DAFPs compared with the DIMFPs after two days at 100\% confluence. Data are presented as the means $\pm \operatorname{SEM}\left(n=3 ;{ }^{*} p<0.05\right)$ 


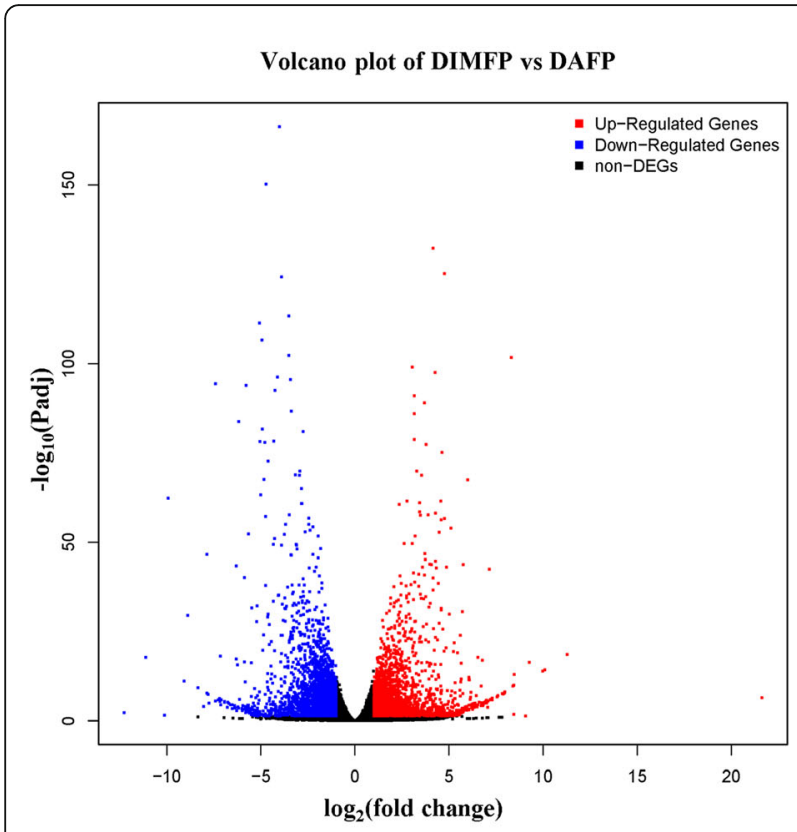

a

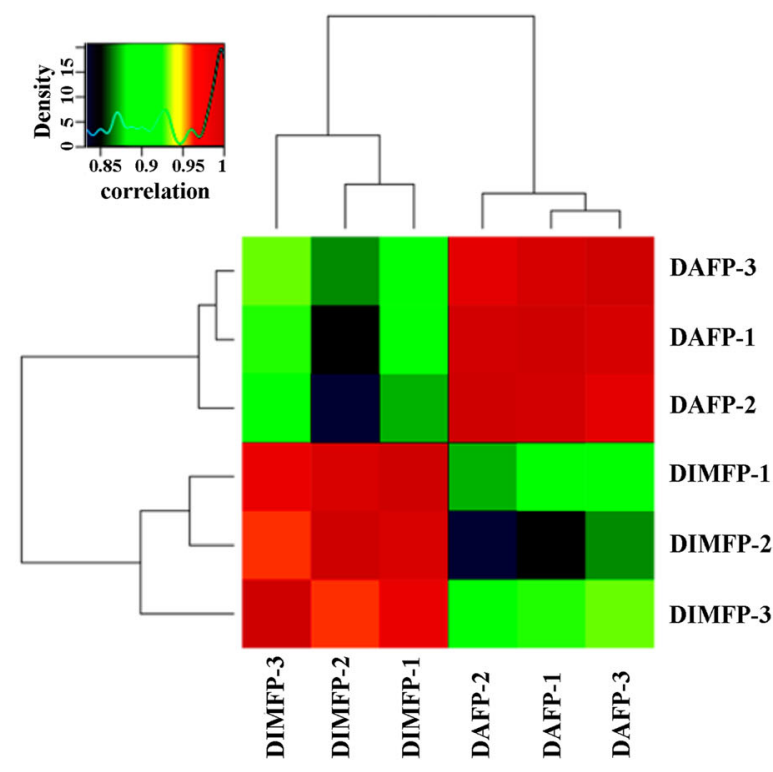

b

Fig. 2 Volcano plot and cluster analysis of differentially expressed genes (DEGs). a Volcano plot. Red dots (UP) represent significantly upregulated genes $\left(\log _{2} F C \geq 1.0, F D R<0.05\right)$; blue dots (DOWN) represent significantly downregulated genes $\left(\log _{2} F C \leq-1.0\right.$, FDR $\left.<0.05\right)$; and black dots $(\mathrm{NO})$ represent DEGs below the level of significance; (b) based on 3486 known DEGs in DIMFPs and DAFPs of chickens, cluster analysis was performed. The results show that the gene expression profiling data in the same group were closely related

differentiation, extracellular matrix, DNA binding, calcium ion binding, etc. (Additional file 3: Table S3). The top 10 terms of each of the biological process (BP), cellular component (CC) and molecular function (MF) terms are shown in Fig. 3.

Meanwhile, 47 pathways were found to be significantly enriched (corrected $P$-value $<0.05$ ) (Additional file 4: Table S4), including some well-known pathways affecting lipid metabolism (PPAR, MAPK, TGF-beta, Wnt, and calcium signaling pathways) and other pathways related to cell communication (focal adhesion, cytokinecytokine receptor interaction, ECM-receptor interaction, tight junction, regulation of the actin cytoskeleton, cell adhesion molecules, and adherens junction pathways). The top 15 enriched pathways are shown in Fig. 4.

\section{DEGs related to lipid metabolism in the two types of preadipocytes}

GO enrichment analysis indicated 72 DEGs related to lipid metabolism, and some representative DEGs were screened (Additional file 5: Table S5). The DEGs related to lipid metabolism were mainly involved in adipocyte differentiation (such as CEBPA, $P P A R G, R B P 7$, and $R X R G$ ), fatty acid transport and fatty acid synthesis (such as ELOVL1, ELOVL6, FABP3, FABP4, FADS6, FADS1 L1, SCD, and SCD5), lipid stabilization (such as CIDEC, PLIN3, PLIN4, and MOGAT1), and lipolysis (such as DGKD, DGKH, $D G K Q$, and $L P L)$. The 20 representative DEGs related to lipid metabolism were randomly selected to validate the gene expression profiling results by qRT-PCR, and the correlation of gene expression profiling and qRT-PCR was analyzed by Spearman rank correlation to confirm the accuracy of the data. The results showed that the fold change in gene expression between the two methods was significantly correlated (Fig. 5a) $(r=0.9666, P<0.01)$.

Among these 20 verified genes, the expression levels of the CEBPA, DGKH, DGKQ, DGKD, FADS1L1, SCD, $S C D 5$, and PPARG genes were significantly $(P<0.05$ or $P<0.01)$ downregulated in DAFPs compared to DIMFPs (Fig. 5b). However, the expression levels of the CIDEC, ELOVL1, ELOVL6, FABP3, FABP4, FADS6, LPL, MOGAT1, PLIN3, PLIN4, RBP7, and RXRG genes were significantly (all $P<0.01$ ) upregulated in DAFPs compared to DIMFPs (Fig. 5c).

\section{Pathways involved in lipid metabolism}

It was found that 19 genes related to lipid metabolism enriched in the PPAR signaling pathway (Additional file 6: Fig. S1). Among these 19 genes, the data from RNA-seq showed that APOA1, ADIPOQ, 


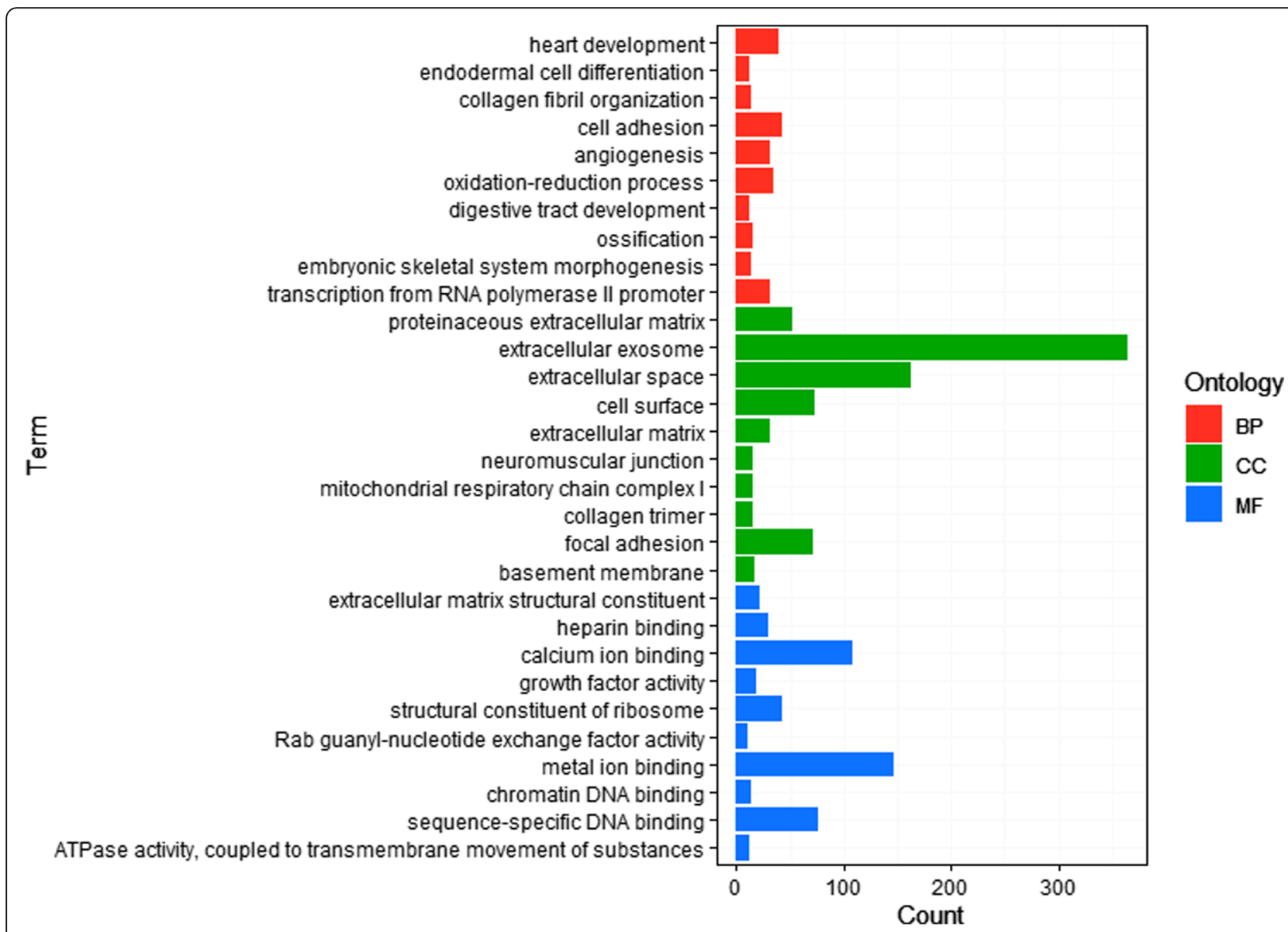

Fig. 3 List of enriched Gene Ontology (GO) terms with the top 10. The enriched Gene Ontology $(G O)$ terms were enriched $(P<0.05)$ based on the $3486 \mathrm{DEGs}$, and the GO terms with the top 10 biological process (BP), cellular component (CC) and molecular function (MF) terms are listed

FABP3, FABP4, FABP7, HMGCS2, LPL and RXRG genes were down-regulated, but ACSL1, FABP5, PCK2, PDPK1, $P P A R G, S C D, S C D 5, S L C 27 A 6$ genes were up-regulated $(P<0.05$ or $P<0.01)$ in the DIMFPs. (Additional file 2: Table S2).

Also, there are a large number of DEGs that were enriched in MAPK- (80 genes), Calcium- (50 genes), and TGF beta (30 genes) signaling pathway, which involved in mediating the biology function of lipid metabolism (Additional file 7: Fig. S2, Additional file 8: Fig. S3, and Additional file 9: Fig. S4). Besides, 245 DEGs also were enriched the pathways related to cell communications (Focal adhesion, Cytokine-cytokine receptor interaction, ECM-receptor interaction, Tight junction, Regulation of actin cytoskeleton, cell adhesion molecules, Adherens junction). However, it was found that the enriched Wnt signaling pathway, as a well-known pathway affecting lipid metabolism, did not medicate the regulation of lipid metabolism. Based on the above information, we proposed the regulatory network for the difference of lipid deposition between chicken DAFPs and DIMFPs (Fig. 6).

\section{Discussion}

Fat has unique distribution characteristics and different economic values in various tissues of animals. In broilers, intramuscular fat is economically desirable in production. Appropriately increased IMF content can improve meat quality, including tenderness, flavor, and juiciness [4-6]. However, excessive abdominal fat deposition has negative impacts on the feed efficiency and carcass yield [2, 3], and decreased abdominal fat deposition is beneficial to reduce waste production and improve consumer acceptance. Lowering abdominal fat and increasing intramuscular fat can effectively increase the economic value of broilers. Therefore, changing the constitution distribution is an important scientific problem for broilers.

Unlike the marbling distribution of IMF in domestic animals, the IMF of chickens cannot be obtained directly from anatomy. Moreover, chicken muscle tissue has a variety of cell compositions [15], and IMF preadipocytes cannot be separated by physical methods due to their similar density to muscle cells. Therefore, high-purity preadipocytes of IMF can only be obtained by the 


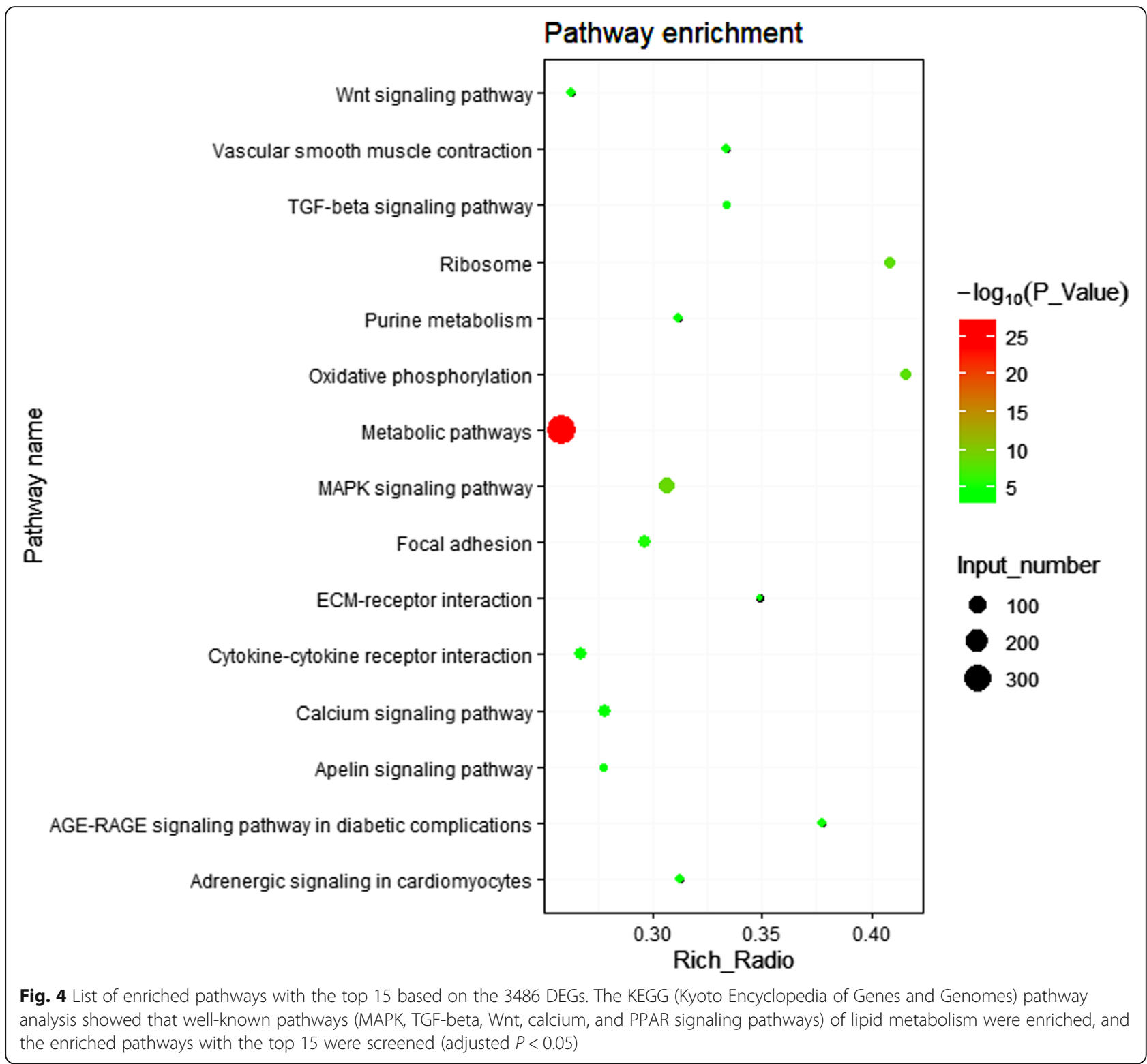

dedifferentiation of mature adipocytes in vitro as described previously [16]. In this study, abdominal fat preadipocytes and intramural preadipocytes were obtained from mature adipocytes of the same chicken to compare their lipogenesis ability under consistent experimental conditions in vitro, establishing a theoretical foundation for the body fat distribution of chickens and providing ideas and development directions for chicken production.

Adipocytes in different tissues are regulated by the adjacent microenvironment to perform the corresponding physiological function [17, 18]. To eliminate the effects of factors in vivo and in vitro, second-generation cells were used. After the cells were overgrown for 2 days, the lipogenesis of adipocytes was detected, which was different from the usual practice of inducing adipocyte differentiation in vitro, avoiding the possibility that the inducers could conceal the lipogenesis of the cells themselves. The results showed that the lipogenesis of preadipocytes derived from abdominal adipocytes was significantly increased compared to that of preadipocytes derived from muscle tissue, consistent with previous in vivo results $[19,20]$, and the increase in the TG content was responsible for the improvement in total lipids.

To identify the regulatory mechanism of lipid deposition for the difference between DIMFPs and DAFPs, RNA sequencing was performed to screen the functional genes and important pathways related to lipid deposition, and quality control by cluster analysis and qRTPCR indicated the reliability of the RNA sequencing 

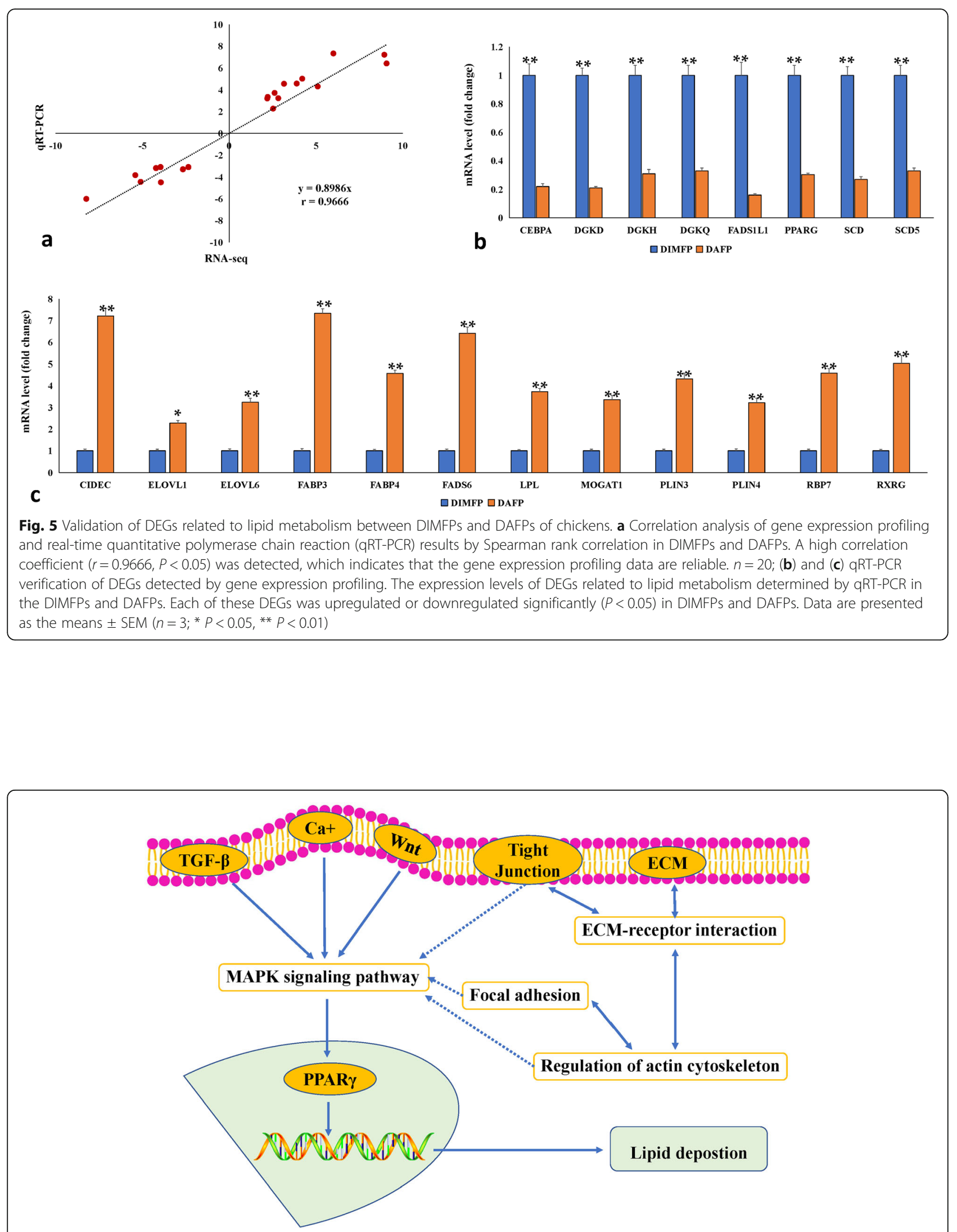

Fig. 6 Proposed regulatory network for the difference in lipid deposition in DIMFPs and DAFPs based on DEGs and enriched signaling pathways 
data. Based on the 3629 screened DEGs, GO terms and KEGG analysis were performed. Forty-seven enriched pathways were screened, including the well-known pathways affecting lipid metabolism (MAPK, TGF beta, Wnt, calcium, and PPAR signaling pathways as well as the pathways related to cell communication). Furthermore, we identified the DEGs related to lipid metabolism according to the enriched GO terms and signaling pathways. Among the 72 DEGs related to lipid metabolism, 19 genes were enriched in the PPAR signaling pathway with the classic mediation of lipid metabolism [21, 22]. Among these 19 genes, the RNA-seq data showed that the APOA1, ADIPOQ, FABP3, FABP4, FABP7, HMGC S2, $L P L$ and RXRG genes were downregulated but the ACSL1, FABP5, PCK2, PDPK1, PPARG, SCD, SCD5, and SLC27A6 genes were upregulated $(P<0.05$ or $P<0.01)$ in the DIMFPs, which had an important regulatory effect on lipid metabolism [14, 21-35]. Therefore, it was considered that these genes and the PPAR signaling pathway had important effects in vitro on regulating the difference in lipid deposition between the DIMFPs and DAFPs of chickens.

It was reported that the MAPK, calcium, and TGF beta signaling pathways interact with the PPAR pathway to regulate lipid metabolism in the lipogenesis process, and a large number of genes were enriched in the MAPK, calcium, and TGF beta signaling pathways [36-38]. Coincidentally, there were a large number of DEGs that were enriched in the MAPK (80 DEGs), calcium (50 DEGs), and TGF-beta (30 DEGs) signaling pathways, which are involved in mediating the biological function of lipid metabolism. According to the enrichment information of these three signaling pathways in this study, the evidence indicated that these three pathways could mediate the biological function of cell differentiation or metabolism. Then, it was deduced that the MAPK, calcium, and TGF beta signaling pathways were also involved in the regulation of lipogenesis between DAFPs and DIMFPs. As in our previous report [39], these pathways related to cell communication also participate in the regulation of lipid deposition through the MAPK signaling pathway in chickens. In this study, multiple enriched pathways related to cell communication (245 DEGs) were screened, including focal adhesion, cytokinecytokine receptor interaction, regulation of the actin cytoskeleton, tight junction, ECM-receptor interaction, and cell adhesion molecules (CAMs), suggesting that the pathways related to cell communication affected the difference in lipid deposition between DIMFPs and DAFPs of chickens. Based on the above information, it was found that the Wnt signaling pathway, a well-known pathway related to lipid metabolism, does not directly regulate lipid metabolism.
It is well known that the fat content in adipose tissue in chickens is far greater than that in intramuscular fat, which may be due to the higher expression of some genes involved in fat synthesis in DAFP than in DIMFP. For example, in our study, the expression of star genes in fat synthetic pathways, such as ELOVL1, ELOVL6, FABP3, FABP4, MOGAT1, PLIN3, and PIN4, which are related to fat synthesis, was significantly increased in DAFP, and the amount of fat synthesized in DAFP was also higher than that in DIMFP, which may be due to the differences in the expression of these genes. Therefore, we speculate that these genes can also be used as biomarkers of fat content. Similarly, these genes may also be used as biomarkers of fat accumulation in chickens, but this requires further experimental verification.

\section{Conclusions}

In brief, our data suggest a difference in lipid deposition between the DIMFPs and DAFPs of chickens in vitro and propose a molecular regulatory network for the difference in lipid deposition between chicken DAFPs and DIMFPs. The lipid content was significantly increased in DAFPs by the direct mediation of PPAR signaling pathways. These findings establish the groundwork and provide new insights into the regulation of tissue-specific fat deposition and optimizing body fat distribution in broilers. In the future, additional studies will be required to complement the effects of these important genes on lipid deposition and pathways in DIMFPs and DAFPs.

\section{Methods}

\section{Animals and ethics statement}

Three BJY chickens were obtained from the Institute of Animal Sciences, CAAS (Beijing, China), which were raised under the same recommended environmental and nutritional conditions. Animal experiments were approved by the Science Research Department, Chinese Academy of Agricultural Sciences (CAAS) (Beijing, China). Three birds were individually euthanized by carbon dioxide anesthesia and exsanguination by severing the carotid artery at 10 days of age, and the pectoralis major and abdominal fat tissues were excised for cell isolation.

\section{Preadipocyte acquisition}

Mature adipocytes from the pectoralis major and abdominal fat tissue were isolated as previously described, and then, preadipocytes were obtained with dedifferentiation treatment as previously described [16]. The abdominal fat tissue and pectoralis major of three chickens were collected and then washed with phosphate-buffered saline (PBS) containing $1 \%$ penicillin-streptomycin (Gibco, Thermo Fisher Scientific Inc., Suzhou, China). The abdominal fat tissue and 
pectoralis major from the same chicken were recorded for the one-to-one correspondence of cell samples in subsequent experiments. After the removal of the blood vessels and connective tissue, the samples were finely minced to $1 \mathrm{~mm}^{3}$ with scissors and then digested in DMEM/F12 (1:1) medium (Gibco, Thermo Fisher Scientific Inc., Suzhou, China) containing 0.1\% Type I collagenase (Sigma-Aldrich, Shanghai, China) in a water bath with continuous shaking at $37^{\circ} \mathrm{C}$ for $60 \mathrm{~min}$. After the termination of digestion and filtration, the cell suspension was centrifuged at $600 \times g$ for $15 \mathrm{~min}$. The top layer containing mature adipocytes was collected and placed in a $25 \mathrm{~cm}^{2}$ cell culture flask, which was inverted and filled with DMEM/F12 (1:1) medium containing $10 \%$ FBS. The floating mature adipocytes adhered to the bottom of the flask and were incubated in a $37^{\circ} \mathrm{C}$ incubator with $5 \% \mathrm{CO}_{2}$. After 3 days, the mature adipocytes would gradually converse to preadipocytes by releasing lipids by exocytosis, the medium was replaced, and the flask was reinverted so that the preadipocytes were on the bottom to proliferate massively. After up to 15 days, the confluence of preadipocytes reached $80-90 \%$, and the cells were subcultured.

\section{Preadipocyte culture and treatment}

The medium was changed every 3 days. After the cells reached $80 \%$ confluence, the cells were passaged, and second passage (P2) preadipocytes were used for further experiments by the dedifferentiation of mature adipocytes from the pectoralis major (DIMFPs) and abdominal fat tissue (DAFPs). Both DIMFPs and DAFPs were plated in 24well or $100-\mathrm{mm}$ dishes, and cells were collected after 2 days at $100 \%$ confluence for further RNA extraction and measurement of biochemical indices (in 100-mm dishes) or Oil Red O staining assays (in 24-well plates).

\section{Oil red $O$ staining assay}

The cellular lipid contents of DIMFPs and DAFPs in 24well plates were determined by Oil Red O staining. The staining steps were as follows: discard the cell culture medium, wash the adherent cells 3 times with PBS, fix the cells with $4 \%$ formalin for $30 \mathrm{~min}$, wash the cells 3 times with PBS, and use Oil Red O (Wuhan AmyJet Scientific Inc., Wuhan, China) dyeing for $60 \mathrm{~min}$. Subsequently, Oil Red $\mathrm{O}$ was discarded, and the cells were washed with PBS 3 times. After the water evaporated, isopropanol was added to extract Oil Red $\mathrm{O}$ for $10 \mathrm{~min}$, and then, the absorbance of the solution was measured at $510 \mathrm{~nm}$ with a Multimode Microplate Reader (Thermo Fisher Scientific Inc., Suzhou, China).

\section{Measurement of biochemical indices}

The two groups of cell samples were homogenized with absolute ethanol at room temperature for $20 \mathrm{~min}$ and centrifuged at $1000 \times g$. Then, the supernatant was collected, and a TG content detection kit (Nanjing Jiancheng Bioengineering Institute, Nanjing, China), TCHO content detection kit (Nanjing Jiancheng Bioengineering Institute, Nanjing, China), and PLIP content detection kit (Beijing Lindemann Biochemical Co., Ltd., Beijing, China) were used to detect the TG, TCHO, and PLIP contents in the two groups of cells. The specific measurement steps were performed according to the manufacturer's instructions.

\section{RNA extraction and identification}

TRIzol reagent (Invitrogen, Carlsbad, CA, USA) was used to extract total RNA from DIMFP cells and DAFP cells, which were cultured in a $100 \mathrm{~mm}$ cell culture dish according to the manufacturer's instructions. The quality of RNA was detected by $1.5 \%$ gel electrophoresis, and the concentration of RNA was determined using a Nanodrop2000 spectrophotometer (Thermo Fisher Scientific Inc., Suzhou, China). The OD260/280 value of all cell samples must be in the range of 1.8 to 2.0 before they can be used. The RNA sample was then used for gene expression analysis.

\section{Gene expression profiling}

High-throughput sequencing (HiSeq2500; Illumina, San Diego, California, USA) was performed on RNA samples of two groups of cells, and the raw data were converted into FASTQ files using bcl2fastq. The clean reads were generated by removing reads with adapter and lowquality sequences and mapped to the reference chicken genome and genes (Gallus gallus, Galgal6; available at https://www.ncbi.nlm.nih.gov/assembly/GCF_ 000002315.6) using TopHat 1.3.2 (https://ccb.jhu.edu/ software/tophat). Gene expression levels were calculated using the RPKM method, as described by Mortazavi et al. [40]. The edgeR $\mathrm{R}$ software package was used to analyze the differentially expressed genes (DEGs) between the DIMFP group and the DAFP group. The DEG screening conditions were $\mid \log _{2}$ FC $\mid \geq 1.0$ and FDR $<0.05$. Cluster analysis of DEGs was performed by the pheatmap software package of $\mathrm{R}$ software.

\section{Gene ontology and Kyoto encyclopedia of genes and genomes analysis}

The ClueGO plug-in and CluePedia plug-in in Cytoscape (https://cytoscape.org/) software were used to perform Gene Ontology (GO) enrichment analysis and functional classification of the DEGs. The significance level of GO term enrichment was set at $P<0.05$. At the same time, the Kyoto Encyclopedia of Genes and Genomes (KEGG) [41] database was used for pathway enrichment analysis of DEGs. $P<0.05$ was considered to be indicative of statistical significance. According to the 
results of GO enrichment analysis and KEGG pathway function enrichment, DEGs related to abdominal fat tissue metabolism were screened.

\section{Real-time quantitative polymerase chain reaction}

A Tiangen ${ }^{\circ}$ FastQuant RT kit (Tiangen, Beijing, China) was used for the reverse transcription of the RNA samples of the two groups of cells according to the steps provided by the manufacturer's instructions, and Primer 5.0 software was used to design specific primers for each gene according to the GenBank sequence (Additional file 10: Table S6). The ABI 7500 real-time PCR detection system (Applied Biosystems, CA, USA) was used for relative quantitative analysis of gene expression in different samples. The total reaction system was $20 \mu \mathrm{L}$ and contained $10 \mu \mathrm{L}$ of SYBR Green mix (TaKaRa, Shanghai, China), $0.5 \mu \mathrm{L}$ of forward primers, and $0.5 \mu \mathrm{L}$ of reverse primers at a concentration of $10 \mathrm{nmol}, 1 \mu \mathrm{L}$ of cDNA, and $8 \mu \mathrm{L}$ of $\mathrm{dd}_{2} \mathrm{O}$. The reaction program was denaturation at $95^{\circ} \mathrm{C}$ for $30 \mathrm{~s}$, followed by 40 cycles of amplification at $95^{\circ} \mathrm{C}$ for $5 \mathrm{~s}$ and $60^{\circ} \mathrm{C}$ for $32 \mathrm{~s}$. The $2^{-\Delta \Delta \mathrm{Ct}}$ method [42] was used to calculate the fold change of gene expression with the expression of actin beta $(\beta$-actin), which was a reference gene.

\section{Statistical analysis}

Three comparison replicates (DIMFPs vs DAFPs) of the cell experiment were set according to the one-to-one correspondence of cell samples from the abdominal fat tissue and pectoralis major of the same chicken. All experiments were repeated three times, and the data obtained are expressed as the mean \pm SEM. Statistically significant differences between the two culture conditions were tested by independent-samples t-tests using SAS 9.2 software (SAS Institute Inc., NC, USA). $P<0.05$ $(*)$ or $P<0.01(* *)$ was considered to be significant. All figures were constructed using GraphPad Prism version 5.02 (GraphPad Software Inc., CA, USA).

\footnotetext{
Abbreviations

ACSL1: Acyl-CoA synthetase long-chain family member 1; APOA1: Apolipoprotein A1; ADIPOQ: Adiponectin, C1Q and collagen domain containing; BP: Biological process; CC: Cellular component; CEBP: CCAAT enhancer binding protein; CIDEC: Cell death inducing DFFA like effector $c$; DEG: Differentially expressed genes; DIMFPs: Dedifferentiated intramuscular preadipocytes; DAFPs: Dedifferentiated abdominal preadipocytes: DGK: Diacylglycerol kinase; ELOVL: Elongase of very long-chain fatty acidslike; FABP: Fatty acid binding protein; FADS: Fatty acid desaturase; HMGC S2: 3-Hydroxy-3-methylglutaryl-coenzyme a synthase 2; IMF: Intramuscular fat; GO: Gene ontology; KEGG: Kyoto encyclopedia of genes and genomes; LPL: Lipoprotein lipase; MAPK: Mitogen- activated protein kinase; MF: Molecular function; MOGAT: Monoacylglycerol O-acyltransferase.; PBS: Phosphate-buffered saline; PCK2: Phosphoenolpyruvate Carboxykinase 2; PDPK1: 3-Phosphoinositide dependent protein kinase-1; PLIN: Perilipin; PPAR: Peroxisome proliferators-activated receptors; qRT-PCR: Quantitative real-time polymerase chain reaction; RBP: Retinol binding protein; RXR: Retinoid X receptor; SCD: Stearoyl-CoA desaturase; SLC27A6: Solute carrier family 27, member 6; Wnt: Wingless/Int
}

\section{Supplementary Information}

The online version contains supplementary material available at https://doi. org/10.1186/s12864-021-07459-8.

Additional file 1: Table S1: Expression of genes (DIMFPS VS DAFPS).

Additional file 2: Table S2: Screened known DEGs (DIMFPS vs DAFPS).

Additional file 3: Table S3: Enriched GO terms based on DEGS (DIMFPS vs DAFPs).

Additional file 4: Table S4: Enriched pathways based on DEGs (DIMFPs vs DAFPs).

Additional file 5: Table S5: Screened DEGs related to lipid metabolism (DIMFPs vs DAFPs).

Additional file 6: Fig. S1: DEGs involved in the PPAR signaling pathway, which were determined based on the 03320 pathway map in the KEGG database. The red box plot shows downregulated genes, and the green box plot shows upregulated genes in the pathway (DIMFPs vs DAFPS). The figure has obtained KEGG copyright permission.

Additional file 7: Fig. S2: DEGs involved in the MAPK signaling pathway, which were determined based on the 04010 pathway map in the KEGG database. The red box plot shows downregulated genes, and the green box plot shows upregulated genes in the pathway (DIMFPs vs DAFPs). The figure has obtained KEGG copyright permission.

Additional file 8: Fig. S3: DEGs involved in the calcium signaling pathway, which were determined based on the 04020 pathway map in the KEGG database. The red box plot shows downregulated genes, and the green box plot shows upregulated genes in the pathway (DIMFPs vs DAFPS). The figure has obtained KEGG copyright permission.

Additional file 9: Fig. S4: DEGs involved in the TGF beta signaling pathway, which were determined based on the 04350 pathway map in the KEGG database. The red box plot shows downregulated genes, and the green box plot shows upregulated genes in the pathway (DIMFPs vs DAFPS). The figure has obtained KEGG copyright permission.

Additional file 10: Table S6: Information on the specific primers used for QRT-PCR.

\section{Acknowledgments}

Not applicable

\section{Authors' contributions}

ZM and NL performed the study, analyzed the data, and drafted the manuscript. $J$ performed the study. LL drafted the manuscript. HXC, HX, and HMK contributed to the design of the study and modifying the manuscript. GPZ and HL designed the study and was in charge of the overall project. All authors submitted comments on drafts, and read and approved the final manuscript.

\section{Funding}

The research was funded by grants from the High-level talent program of Foshan University (No. cgz07243), Key-Area Research and Development Program of Guangdong Province (No. 2020B020222002), Innovation Team of Precise Animal Breeding (No. 2019KCXTD004), and Guangdong Provincial Key Laboratory of Animal Molecular Design and Precise Breeding (No.

2019B030301010, No. 201KSYS011). The funding agencies had not involved in the experimental design, analysis, and interpretation of the data or writing of the manuscript.

\section{Availability of data and materials}

All of raw data generated during the study are uploaded to the NCBI-SRA database (https://www.ncbi.nlm.nih.gov/sra/) under the accession number SRR13665175, SRR13665173, SRR13665172, SRR13665171, SRR13665170, SRR13665174.

\section{Declarations}

\section{Ethics approval and consent to participate}

This study was conducted by the Guidelines for Experimental Animals established by the Ministry of Science and Technology (Beijing, China). All 
experimental protocols were approved by the Foshan University, Animal Ethics Approval Committee under the No. 2019GA19.

\section{Consent for publication}

Not applicable.

\section{Competing interests}

The authors declare that they have no competing interests.

\section{Author details}

'School of Life Science and Engineering, Foshan University; Guangdong Provincial Key Laboratory of Animal Molecular Design and Precise Breeding, Foshan 534861, China. ${ }^{2}$ Institute of Animal Sciences, Chinese Academy of Agricultural Sciences; State Key Laboratory of Animal Nutrition, Beijing 100193, China.

Received: 22 June 2020 Accepted: 19 February 2021 Published online: 03 April 2021

\section{References}

1. Ma JS, Chang WH, Liu GH, Zhang S, Zheng AJ, Li Y, et al. Effects of flavones of sea buckthorn fruits on growth performance, carcass quality, fat deposition and Lipometabolism for broilers. Poult Sci. 2015;94(11):2641-9.

2. Demeure O, Duclos MJ, Bacciu N, Le Mignon G, Filangi O, Pitel F, et al. Genome-wide interval mapping using SNPs identifies new QTL for growth, body composition and several physiological variables in an F2 intercross between fat and lean chicken lines. Genet Sel Evol. 2013;45:36.

3. Ramiah SK, Meng GY, Sheau WT, Swee KY, Ebrahimi M. Dietary conjugated linoleic acid supplementation leads to Downregulation of PPAR transcription in broiler chickens and reduction of adipocyte cellularity. PPAR Res. 2014;2014:137652.

4. Nishimura T. The role of intramuscular connective tissue in meat texture Anim Sci J. 2010;81(1):21-7.

5. Ge K, Ye P, Yang L, Kuang J, Chen X, Geng Z. Comparison of slaughter performance, meat traits, serum lipid parameters and fat tissue between Chaohu ducks with high- and low-intramuscular fat content. Anim Biotechnol. 2020;31(3):245-55.

6. Frank D, Watkins P, Ball A, Krishnamurthy R, Piyasiri U, Sewell J, et al. Impact of brassica and lucerne finishing feeds and intramuscular fat on lamb eating quality and flavor. A cross-cultural study using Chinese and non-Chinese Australian consumers. J Agric Food Chem. 2016;64(36):6856-68.

7. Starkey CP, Geesink GH, Collins D, Hutton Oddy V, Hopkins DL. Do sarcomere length, collagen content, $\mathrm{pH}$, intramuscular fat and desmin degradation explain variation in the tenderness of three ovine muscles? Meat Sci. 2016;113:51-8.

8. Chu W, Wei W, Han H, Gao Y, Liu K, Tian Y, et al. Muscle-specific downregulation of GR levels inhibits adipogenesis in porcine intramuscular adipocyte tissue. Sci Rep. 2017;7(1):510.

9. Kouba M, Bonneau M. Compared development of intermuscular and subcutaneous fat in carcass and primal cuts of growing pigs from 30 to 140kg body weight. Meat Sci. 2009;81(1):270-4.

10. Arrighi N, Moratal C, Clément N, Giorgetti-Peraldi S, Peraldi P, Loubat A, et al. Characterization of adipocytes derived from fibro/adipogenic progenitors resident in human skeletal muscle. Cell Death Dis. 2015;6(4): e1733.

11. Jiang S, Wei H, Song T, Yang Y, Peng J, Jiang S. Transcriptome comparison between porcine subcutaneous and intramuscular stromal vascular cells during adipogenic differentiation. PLoS One. 2013;8(10):e77094.

12. Wang S, Zhou G, Shu G, Wang L, Zhu X, Gao P, et al. Glucose utilization, lipid metabolism and BMP-Smad signaling pathway of porcine intramuscular preadipocytes compared with subcutaneous preadipocytes. Cell Physiol Biochem. 2013;31(6):981-96.

13. Hausman GJ, Poulos S. Recruitment and differentiation of intramuscular preadipocytes in stromal-vascular cell cultures derived from neonatal pig semitendinosus muscles. J Anim Sci. 2004;82(2):429-37.

14. Zhang M, Li F, Ma XF, Li WT, Jiang RR, Han RL, et al. Identification of differentially expressed genes and pathways between intramuscular and abdominal fat-derived Preadipocyte differentiation of chickens in vitro. BMC Genomics. 2019;20(1):743.

15. Hocquette JF, Gondret F, Baeza E, Medale F, Jurie C, Pethick DW. Intramuscular fat content in meat-producing animals: development, genetic and nutritional control, and identification of putative markers. Animal. 2010; 4:303-19.

16. Cui HX, Guo LP, Zhao GP, Liu RR, Li QH. Method using a co-culture system with high-purity intramuscular preadipocytes and satellite cells from chicken pectoralis major muscle. Poult Sci. 2018;97(10):3691-7.

17. Zhou G, Wang S, Wang Z, Zhu X, Shu G, Liao W, Yu K, Gao P, Xi Q, Wang XJ. Global comparison of gene expression profiles between intramuscular and subcutaneous adipocytes of neonatal landrace pig using microarray. Meat Sci. 2010;86(2):440-50

18. Sun WX, Wang HH, Jiang BC, Zhao YY, Xie ZR, Xiong K, Chen J. Global comparison of gene expression between subcutaneous and intramuscular adipose tissue of mature Erhualian pig. Genet Mol Res. 2013;12(4):5085-101.

19. Mérida I, Ávila-Flores A, Merino E. Diacylglycerol kinases: at the hub of cell signalling. Biochem J. 2008;409(1):1-18.

20. Sakane F, Mizuno S, Takahashi D, Sakai H. Where do substrates of diacylglycerol kinases come from Diacylglycerol kinases utilize diacylglycerol species supplied from phosphatidy-linositol turnover-independent pathways. Adv Biol Regul. 2018;67:101-8.

21. Lee $Y E$, He HL, Shiue $Y L$, Lee $S W$, Lin LC, Wu TF, et al. The prognostic impact of lipid biosynthesis-associated markers, HSD17B2 and HMGCS2, in rectal cancer treated with neoadjuvant concurrent chemoradiotherapy. Tumour Biol. 2015;36(10):7675-83.

22. Pazhouhandeh M, Sahraian MA, Siadat SD, Fateh A, Vaziri F, Tabrizi F, et al. A systems medicine approach reveals disordered immune system and lipid metabolism in multiple sclerosis patients. Clin Exp Immunol. 2018; 192(1):18-32.

23. Lefterova MI, Haakonsson AK, Lazar MA, Mandrup S. PPARy and the global map of adipogenesis and beyond. Trends Endocrinol Metab. 2014;25(6): 293-302.

24. Capobianco E, Martínez N, Fornes D, Higa R, Di Marco I, Basualdo MN, et al. PPAR activation as a regulator of lipid metabolism, nitric oxide production and lipid peroxidation in the placenta from type 2 diabetic patients. Mol Cell Endocrinol. 2013;377(1-2):7-15.

25. Tian W, Wang D, Wang Z, Jiang K, Li Z, Tian Y, et al. Evolution, expression profile, and regulatory characteristics of ACSL gene family in chicken (Gallus gallus). Gene. 2021;764:145094.22.

26. Cryer A. Tissue lipoprotein lipase activity and its action in lipoprotein metabolism. Int J BioChemiPhysics. 1981;13:525-41.

27. Mihelic R, Winter H, Powers JB, Das S, Lamour K, Campagna SR, et al. Genes controlling polyunsaturated fatty acid synthesis are developmentally regulated in broiler chicks. Br Poult Sci. 2020;61(5):508-17.

28. Wu X, Zou X, Chang Q, Zhang Y, Li Y, Zhang L, et al. The evolutionary pattern and the regulation of stearoyl-CoA desaturase genes. Biomed Res Int. 2013;2013:856521.

29. Hughes AL, Piontkivska H. Evolutionary diversification of the avian fatty acidbinding proteins. Gene. 2011;490(1-2):1-5.

30. Jin S, Lee SH, Lee DH, Manjula P, Lee SH, Lee JH. Genetic association of DEGS1, ELOVL6, FABP3, FABP4, FASN and SCD genes with fatty acid composition in breast and thigh muscles of Korean native chicken. Anim Genet. 2020;51(2):344-5.

31. Li J, Xing S, Zhao G, Zheng M, Yang X, Sun J, et al. Identification of diverse cell populations in skeletal muscles and biomarkers for intramuscular fat of chicken by single-cell RNA sequencing. BMC Genomics. 2020;21(1):752.

32. Liu L, Liu X, Cui H, Liu R, Zhao G, Wen J. Transcriptional insights into key genes and pathways controlling muscle lipid metabolism in broiler chickens. BMC Genomics. 2019;20(1):863.

33. Nafikov RA, Schoonmaker JP, Korn KT, Noack K, Garrick DJ, Koehler KJ, et al. Association of polymorphisms in solute carrier family 27, isoform A6 (SLC27A6) and fatty acid-binding protein-3 and fatty acid-binding protein-4 (FABP3 and FABP4) with fatty acid composition of bovine milk. J Dairy Sci. 2013;96(9):6007-21.

34. Loor JJ, Dann HM, Everts RE, Oliveira R, Green CA, Guretzky NA, et al. Temporal gene expression profiling of liver from periparturient dairy cows reveals complex adaptive mechanisms in hepatic function. Physiol Genomics. 2005;23(2):217-26.

35. Montserrat A, Kersten S. Regulation of lipid droplet-associated proteins by peroxisome proliferator-activated receptors. Biochim Biophys Acta Mol Cell Biol Lipids. 2017;1862(10 Pt B):1212-20.

36. He YH, He Y, Liao XL, Niu YC, Wang G, Zhao C, et al. The calcium-sensing receptor promotes adipocyte differentiation and adipogenesis through PPARy pathway. Mol Cell Biochem. 2012;361(1-2):321-8. 
37. Han H, Cao A, Wang L, Guo H, Zang Y, Li Z, et al. Huangqi decoction ameliorates streptozotocin-induced rat diabetic nephropathy through antioxidant and regulation of the TGF- $\beta$ /MAPK/PPAR- $\gamma$ signaling. Cell Physiol Biochem. 2017:42(5):1934-44.

38. Agarwal S, Chattopadhyay M, Mukherjee S, Dasgupta S, Mukhopadhyay S, Bhattacharya S. Fetuin-A downregulates adiponectin through Wnt-PPARY pathway in lipid induced inflamed adipocyte. Biochim Biophys Acta Mol basis Dis. 2017;1863(1):174-81.

39. Cui H-X, Liu R-R, Zhao G-P, Zheng M-Q, Chen J-L, Wen J. Identification of differentially expressed genes and pathways for intramuscular fat deposition in pectoralis major tissues of fast-and slow-growing chickens. BMC Genomics. 2012;13:213.

40. Mortazavi A, Williams BA, McCue K, Schaeffer L, Wold B. Mapping and quantifying mammalian transcriptomes by RNA-Seq. Nat Methods. 2008; 5:621.

41. Kanehisa M, Goto S. KEGG: Kyoto encyclopedia of genes and genomes. Nucleic Acids Res. 1999;28(1):27-30.

42. Livak KJ, Schmittgen TD. Analysis of relative gene expression data using real-time quantitative PCR and the $2^{-\Delta \Delta C T}$ method. Methods Companion Methods Enzymol. 2001;25(4):402-8.

\section{Publisher's Note}

Springer Nature remains neutral with regard to jurisdictional claims in published maps and institutional affiliations.

Ready to submit your research? Choose BMC and benefit from:

- fast, convenient online submission

- thorough peer review by experienced researchers in your field

- rapid publication on acceptance

- support for research data, including large and complex data types

- gold Open Access which fosters wider collaboration and increased citations

- maximum visibility for your research: over $100 \mathrm{M}$ website views per year

At BMC, research is always in progress.

Learn more biomedcentral.com/submissions 